\title{
Transthyretin Exerts Pro-Apoptotic Effects in Human Retinal Microvascular Endothelial Cells Through a GRP78- Dependent Pathway in Diabetic Retinopathy
}

\author{
Jun Shao ${ }^{a}$ Ying Yin ${ }^{a} \quad$ Xiaowen Yin ${ }^{a}$ Li Jia Yu Xin ${ }^{b}$ Jian Zou ${ }^{c}$ Yong Yao ${ }^{a}$ \\ a Department of Ophthalmology, Wuxi People's Hospital affiliated to Nanjing Medical University, Wuxi, \\ Jiangsu, ${ }^{b}$ School of Biotechnology, Jiangnan University, Key Laboratory of Industry Biotechnology, \\ Ministry of Education, Wuxi, Jiangsu, ${ }^{c}$ Center of Clinical Research, Wuxi People's Hospital affiliated to \\ Nanjing Medical University, Wuxi Institute of Translational Medicine, Wuxi, Jiangsu, PR China
}

\section{Key Words}

Transthyretin - Diabetic retinal $\cdot$ Human retinal microvascular endothelial cells - Apoptosis • GRP78-depedent pathway

\begin{abstract}
Background/Aims: Diabetic retinopathy (DR) is one of the main causes of blindness in the world. Our previous study showed that transthyretin (TTR) regulates key genes in the Tie2 pathway and inhibits the development of neovascularization in DR, but the mechanism is still unclear. Here, we investigated how TTR affects the progression of neovascularization in DR. Methods: Natural and simulated DR media (hyperglycemia and hypoxia) were used to culture human retinal microvascular endothelial cells (hRECs). Flow cytometry was employed to investigate the effect of TTR on apoptosis of hRECs. Fluorescent labeling and immunofluorescence staining were used to determine the TTR distribution in hRECs. The membrane proteins of hRECs were extracted and applied to a sepharose-TTR column, and the captured proteins were identified by Mass Spectrometric analysis. Gene knock-down and western blotting assays were used to study the key signal pathway of the most abundant identified protein. Results: TTR induced apoptosis of hRECs in an environment that simulated hypoxia. Immunofluorescent staining showed that TTR could enter the nuclei of hRECs. A total of 30 unique TTR-captured proteins were identified by Mass Spectrometry, and glucoseregulated protein 78 (GRP78) was one of the most abundant. Western blotting and gene knock-down indicated that TTR might upregulate GRP78 and facilitate apoptosis through the eIF2 $\alpha / C H O P$ pathway. Conclusions: In the DR environment (hyperglycemia and hypoxia), TTR was shown to repress neovascularization by promoting apoptosis of hRECs through a GRP78dependent pathway.




\section{Cellular Physiology Cell Physiol Biochem 2017;43:788-800

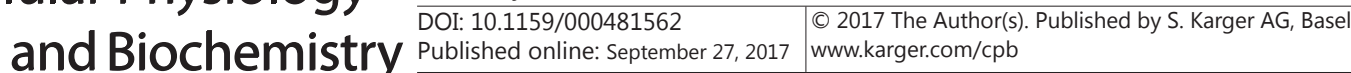 \\ Shao et al.: TTR Induces HRECs Apoptosis Through GRP78-Pathway in DR}

\section{Introduction}

Diabetic retinopathy (DR) is one of the most serious diabetic microangiopathic complications and is one of leading cause of blindness in the world [1]. An epidemiological survey showed that the prevalence rate of DR in China has increased on a yearly basis [2]. DR is caused by alterations in the retinal micro vasculature in diabetic patients, and retina neovascularization is one of the most significant clinical properties of proliferative diabetic retinopathy (PDR) [3]. Therefore, anti-neovascularization strategies are the only effective form of therapy for PDR. However, this therapeutic strategy cannot fundamentally reverse the patient's permanently damaged vision. Thus, optimized methods are urgently needed to investigate detailed mechanisms of DR and to deliver potent treatments against DR [4]. Recently, some new mechanisms have been reported in the development of DR, including protein palmitoylation [5] and autophagy [6]. In addition, several compounds and potential targets have been proven to attenuate neovascularization and retinal vascular leakage through specific pathways $[7,8]$. A previous report indicated that retinal hypoxia is a major component of the pathophysiology in the advanced, sight-threatening stages of DR [9]. Under hypoxic conditions, hypoxiainducible factor- $1 \alpha$ (HIF- $1 \alpha$ ) is activated and stabilized. This factor regulates hypoxiainducible genes including VEGFA which is involved in the angiogenic response, as well as erythropoiesis factor (e.g., EPO) [10-12]. Thus, physiological and pathological research on hRECs in a hypoxic environment is helpful in understanding the development of DR.

Transthyretin (TTR) is a 55-kDa homotetrameric protein that is found in the plasma and cerebrospinal fluid. This protein is synthesized in the liver (blood source) and choroid plexus (cerebrospinal fluid source). It is also produced by human retinal pigment epithelial cells (hRPECs) in ocular tissue [13]. The physiological functions of TTR are to transport thyroxine and retinol through retinol-binding protein (RBP). In ophthalmology research, various eye diseases are associated with TTR. Nuclear cataracts are associated with low protein intake and low serum levels of TTR [14]. Immunohistochemical analysis of the drusen in patients with age-related macular degeneration revealed the presence of TTR [15]. In our previous study, patients with macular detachment (MD) and macular holes (MH) demonstrated complete or partial losses of the natural bio-functions of the vitreous TTR. However, these losses appeared to be associated with abnormally high TTR levels [16]. The TTR in the MD vitreous compartment had an abnormally stable tetrameric structure, possibly due to misfolding [17]. In our recent studies, we found that the key genes in Tie2 pathway for DR neovascularization, including Tie2 (tyrosine-protein kinase receptor 2), VEGFR1 (vascular endothelial growth factor receptor 1), VEGFR2 (vascular endothelial growth factor receptor 2), Angpt1 (Angiopoietin 1) and Angpt2 (Angiopoietin 2), were regulated by TTR [18, 19]. TTR represses neovascularization in DR, but the mechanisms by which TTR affects the development of neovascularization in DR remains unclear.

In the present study, we elucidated the molecular pathways that involved TTR under DR, during both physiologic and pathologic conditions. Proteomic assays were used to investigate the specific mechanisms by which TTR efficiently inhibits neovascularization in DR. Membrane proteins were applied to an immobilized-TTR column and the TTRcaptured proteins were identified with LTQ-MS analysis. GRP78 was identified as the key hub stimulated by TTR, and the correlated interaction clusters were investigated herein. Our study revealed that TTR promotes apoptosis of hRECs via a GRP78-dependent pathway in simulated DR environments.

\section{Materials and Methods}

Reagents and cell culture

This study followed the tenets of the Declaration of Helsinki and the ARVO statement for research involving human subjects and was approved by the Ethics Committee of Nanjing Medical University. The Expresso Rhamnose kit was purchased from Lucigen (Middleton, WI, 


\section{Cellular Physiology Cell Physiol Biochem 2017;43:788-800 \begin{tabular}{l|l} 
and Biochemistry $10.1159 / 000481562$ & $\begin{array}{l}\text { O } 2017 \text { The Author(s). Published by S. Karger AG, Basel } \\
\text { www.karger.com/cpb }\end{array}$
\end{tabular} \\ Shao et al.: TTR Induces HRECs Apoptosis Through GRP78-Pathway in DR}

USA). hRECs were purchased from Shanghai Bioleaf Biotech Co. (Shanghai, China). Dulbecco's Modified Eagle's medium (DMEM), fetal bovine serum (FBS), PBS $(1 \times ; 155.2 \mathrm{mM} \mathrm{NaCl}, 2.7 \mathrm{mM}$ $\mathrm{Na}_{2} \mathrm{HPO}_{4}, 1.5 \mathrm{mM} \mathrm{KH} \mathrm{PO}_{4}, \mathrm{pH} 7.4$ ) and Lipofectamine-2000 were from Life Technologies (Carlsbad, CA, USA). CoCl,$N$-(5-Fluoresceinyl) maleimide and glucose were from Sigma (St. Louis, MO, USA). The Mem-PER ${ }^{\mathrm{TM}}$ PLUS Kit, Dead Cell Apoptosis Kit and Bicinchoninic acid (BCA) assay kit were from ThermoFisher Scientific (Rockford, IL, USA). CNBr-activated sepharose was from GE Healthcare (Uppsala, Sweden). The ToxinEraser ${ }^{\mathrm{TM}}$ Endotoxin Removal kit was from GenScript (Piscataway, NJ, USA). The GRP78 siRNA was from Santa Cruz Biotechnology (Santa Cruz, CA, USA). Anti-TTR and -GRP78 antibodies were from Abcam Trading Company (Shanghai, China). Antibodies against CHOP, ATF4 and PERK were from Proteintech Group (Rosemont, IL, USA). Antibodies to eIF2 $\alpha$ were obtained from Cell Signaling Technology (Danvers, MA, USA). Secondary antibodies including Alexa Fluor 488 donkey anti-mouse IgG antibodies (1:500-1000) and Alexa Fluor 594 donkey anti-rabbit IgG antibodies (1:500-1000) were from Life Technologies (Carlsbad, CA, USA). Other reagents and chemicals were purchased from local companies and were of superior analytical grades.

\section{Expression, preparation and labeling of TTR}

The wild-type TTR was produced in an E. coli expression system from Lucigen (Middleton, WI, USA) and purified as described previously [20]. The human TTR cDNA was inserted into a pRham ${ }^{\text {TM }} \mathrm{N}$-HIS vector, and the plasmid was used to transform E. coli BL21 (DE3). The E. coli BL21 (DE3) cells containing the plasmid were grown overnight in $5 \mathrm{~mL}$ LB broth with $50 \mu \mathrm{g} /$ $\mathrm{mL}$ ampicillin at $37^{\circ} \mathrm{C}$ and $200 \mathrm{rpm}$. A flask containing $500 \mathrm{~mL}$ of culture medium (LB broth with ampicillin) was inoculated with $5 \mathrm{~mL}$ of the starter cultures at $37^{\circ} \mathrm{C}$ and $200 \mathrm{rpm}$ until the $A_{600 \mathrm{~nm}}$ reached 0.6 . The expression of TTR was then induced with $0.2 \%$ rhamnose for $5-6$ $\mathrm{h}$. The cells were collected by centrifugation at $4{ }^{\circ} \mathrm{C}$ and $8000 \times \mathrm{g}$ for $30 \mathrm{~min}$, and then the cell pellet was disrupted by sonication. After centrifugation at $4{ }^{\circ} \mathrm{C}$ and $8000 \times \mathrm{g}$ for $30 \mathrm{~min}$, the supernatant was applied to a Ni-Sepharose column, which was subsequently washed with washing buffer 1 ( $20 \mathrm{mM}$ Tris- $\mathrm{HCl}, \mathrm{pH} 8.0,20 \mathrm{mM}$ imidazole), washing buffer 2 (20 mM Tris-HCl, pH 8.0, $50 \mathrm{mM}$ imidazole) and washing buffer 3 (20 mM Tris- $\mathrm{HCl}, \mathrm{pH}$ 8.0, $100 \mathrm{mM}$ imidazole). The captured TTR was finally eluted with elution buffer $(20 \mathrm{mM}$ Tris- $\mathrm{HCl}, \mathrm{pH} 8.0$, $400 \mathrm{mM}$ imidazole). The imidazole was depleted by 6 cycles of ultrafiltration in an Amicon Ultra YM-3 tube (Merck Millipore). The endotoxin in the protein sample was depleted using a ToxinEraser ${ }^{\mathrm{TM}}$ Endotoxin Removal kit from GenScript. The resulting samples were subjected to SDS-PAGE analysis.

The only cysteine in TTR is located at the N-terminus, therefore, TTR could be site-specifically labeled at this cysteine with $\mathrm{N}$-(5-Fluoresceinyl) maleimide as previously reported $[21,22]$. The purified TTR was incubated with $5 \mathrm{mM}$ DTT in $20 \mathrm{mM}$ Tris- $\mathrm{HCl}(\mathrm{pH} 8.0)$ at 4 ${ }^{\circ} \mathrm{C}$ for $2 \mathrm{~h}$, and then, to remove the DTT, the protein was precipitated and washed with $75 \%$ $\left(\mathrm{NH}_{4}\right)_{2} \mathrm{SO}_{4}$ at $4{ }^{\circ} \mathrm{C}$. Then, the protein was reacted with $\mathrm{N}$-(5-Fluoresceinyl) maleimide at $4{ }^{\circ} \mathrm{C}$ overnight at a ratio of $1: 5$. After this reaction, the free labeling reagent was removed by 6 cycles of ultrafiltration.

Culture of hRECs in natural and simulated DR environments

hRECs from passages 3-6 were cultured in 6-well plates using the following protocols. Low glucose, high glucose, and hypoxic media were prepared according to previous reports $[18,19]$ (Table 1). For the natural medium (LG), cells

Table 1. Details of hRECs culture media. Abbreviations: LG, Low glucose; HG, hyperglycemia; TTR, transthyretin; DMEM, Dulbecco's Modified Eagle's medium (DMEM); FBS, fetal bovine serum

\begin{tabular}{lccccc}
\hline Medium & DMEM & FBS $(\%)$ & Glucose $(\mathrm{mM})$ & $\mathrm{CoCl}_{2}(\mu \mathrm{M})$ & $\mathrm{TTR}(\mu \mathrm{M})$ \\
\hline LG & + & 10 & 5.5 & - & - \\
LG+TTR & + & 10 & 5.5 & - & 4 \\
LG+Hypoxia & + & 10 & 5.5 & 200 & - \\
LG+Hypoxia+TTR & + & 10 & 5.5 & 200 & 4 \\
HG & + & 10 & 25 & - & - \\
HG+TTR & + & 10 & 25 & - & 4 \\
HG+Hypoxia & + & 10 & 25 & 200 & - \\
HG+Hypoxia+TTR & + & 10 & 25 & 200 & 4 \\
\hline
\end{tabular}




\section{Cellular Physiology Cell Physiol Biochem 2017;43:788-800 \begin{tabular}{l|l} 
DOI: 10.1159/000481562 & $\begin{array}{l}\text { O } 2017 \text { The Author(s). Published by S. Karger AG, Basel } \\
\text { www.karger.com/cpb }\end{array}$
\end{tabular} \\ Shao et al.: TTR Induces HRECs Apoptosis Through GRP78-Pathway in DR}

were cultured at $37^{\circ} \mathrm{C}$ in a Series $8000 \mathrm{WJ}$ cell incubator (ThermoScientific,Waltham, MA, USA) in DMEM containing $5.5 \mathrm{mM}$ glucose and 10\% FBS. To simulate the hyperglycemia (HG) of a DR environment, the glucose in DMEM was increased to $25 \mathrm{mM}$; hypoxia was induced with $200 \mu \mathrm{M} \mathrm{CoCl}_{2}$ (LG+Hypoxia and $\mathrm{HG}+$ Hypoxia) [23-25].

\section{Flow cytometric analysis}

hRECs from passage 4 were diluted to $8 \times 10^{4} / \mathrm{mL}$ and incubated in 6-well plates with low glucose media for approximately $12 \mathrm{~h}$ until confluent. After washing with PBS, the cells were further cultured for $48 \mathrm{~h}$ at $37^{\circ} \mathrm{C}$ in low glucose (LG), low glucose with hypoxia (LG+Hypoxia), high glucose (HG), or high glucose with hypoxia (HG+Hypoxia) media. Approximately $4 \mu \mathrm{mol} / \mathrm{L}$ TTR was included in the media according to a previously described protocol $[18,19]$. The cells were trypsinized, collected, washed in PBS, and analyzed using a Dead Cell Apoptosis Kit (ThermoFisher Scientific). The cells were resuspended in $100 \mu \mathrm{L}$ of binding buffer containing $5 \mu \mathrm{L}$ Annexin V-FITC and $5 \mu \mathrm{L}$ propidium iodide. The samples were mixed gently and incubated at room temperature in the dark for $20 \mathrm{~min}$. An additional $500 \mu \mathrm{L}$ binding buffer was then added to each sample tube, and the samples were analyzed using fluorescence-activated cell sorting (FACS) (BeckmanCoulter, Kraemer Boulevard Brea, CA, USA). A minimum of 10, 000 cells within the gated region were collected. All the tests were repeated three times.

\section{Membrane protein extraction}

The membrane proteins from hRECs (passage 5) were extracted using Mem-PER ${ }^{\mathrm{TM}}$ PLUS Kit. A total of $5 \times 10^{6}$ cells were resuspended in the growth media by scraping the cells off the surface of the plate with a cell scraper. The harvested cell suspension was centrifuged at $300 \times \mathrm{g}$ for $5 \mathrm{~min}$. The cell pellet was washed with $3 \mathrm{~mL}$ Cell Wash Solution and centrifuged at $300 \times \mathrm{g}$ for $5 \mathrm{~min}$, after which the supernatant was carefully removed and discarded. The cells were resuspended in $1.5 \mathrm{~mL}$ Cell Wash Solution, transferred to a $2 \mathrm{~mL}$ centrifuge tube and centrifuged at $300 \times \mathrm{g}$ for $5 \mathrm{~min}$. The supernatant was discarded, and $0.75 \mathrm{~mL}$ Permeabilization Buffer was added to the cell pellet. After vortexing briefly, the sample was incubated for $10 \mathrm{~min}$ at $4{ }^{\circ} \mathrm{C}$ with constant mixing, and then centrifuged for 15 $\min$ at $16,000 \times \mathrm{g}$. The supernatant, which contained the cytosolic proteins, was carefully removed and transferred to a new tube. The pellet was resuspended in $0.5 \mathrm{~mL}$ Solubilization Buffer by trituration. The tubes were incubated at $4{ }^{\circ} \mathrm{C}$ for $30 \mathrm{~min}$ with constant mixing and then centrifuged at $16,000 \times \mathrm{g}$ for $15 \mathrm{~min}$ at $4{ }^{\circ} \mathrm{C}$. The supernatant containing the solubilized membrane and membrane-associated proteins was transferred to a new tube. The resulting fractions were stored on ice or stored as aliquots at $-80^{\circ} \mathrm{C}$ for future use.

\section{Screening for TTR-associated proteins}

Recombinant human TTR was immobilized on CNBr-activated sepharose beads by combining $2 \mathrm{mg}$ TTR with $1 \mathrm{~mL}$ beads in $0.1 \mathrm{M} \mathrm{NaHCO}_{3} / 0.5 \mathrm{M} \mathrm{NaCl}$ (pH 8.3). After mixing at $4{ }^{\circ} \mathrm{C}$ overnight, the beads were packed into a $1 \mathrm{~mL}$ column and equilibrated with $20 \mathrm{mM}$ PBS containing $0.1 \%$ Triton $\mathrm{X}-100(\mathrm{pH} 7.2)$. The extracted membrane proteins were applied to the TTR-sepharose column, washed five times with $20 \mathrm{mM}$ PBS/0.1 Triton X-100 (pH 7.2), and the proteins captured by TTR were eluted with $20 \mathrm{mM} \mathrm{PBS} / 0.1$ Triton X-100/0.5 M NaCl (pH 7.2).

The eluted proteins were analyzed using LTQ-MS. Briefly, $25 \mu \mathrm{g}$ trypsin was dissolved in $2.5 \mathrm{~mL}$ tosylphenylalanylchloromethane (TCPK) mixed with $250 \mu \mathrm{L}$ of $0.1 \%$ redistilled acetonitrile. A $15 \mu \mathrm{L}$ sample of this trypsin solution was activated in $100 \mu \mathrm{L} 50 \mathrm{mM} \mathrm{NH}_{4}$ $\mathrm{HCO}_{3}$. A $5 \mu \mathrm{L}$ aliquot of the TTR-captured proteins was reduced with $100 \mu \mathrm{L}$ of reducing buffer $(200 \mu \mathrm{L}$ TCEP in $2 \mathrm{~mL}$ digestion buffer ( $50 \mathrm{mM}$ ammonium bicarbonate, $\mathrm{pH}$ 7.5)) and incubated at $60{ }^{\circ} \mathrm{C}$ for $10 \mathrm{~min}$. Following this incubation, $100 \mu \mathrm{L}$ of alkylation buffer $(60$ $\mathrm{mg}$ iodoacetamide in $3 \mathrm{~mL}$ digestion buffer) was added to the tube, and the mixture was incubated in the dark at room temperature for $1 \mathrm{~h}$ for carboxymethylation and oxidation of the cysteine and methionine residues. Then, $20 \mu \mathrm{L}$ of the activated trypsin solution was 
added to the tube and incubated at $37^{\circ} \mathrm{C}$ for $1 \mathrm{~h}$, followed by incubation at $25^{\circ} \mathrm{C}$ overnight with gentle mixing. This peptide mixture was injected onto a Zorbax300SB-C18 peptide trap (Agilent Technologies, Wilmington, DE, USA) for desalting, and separation was performed on a Zorbax 300SB-C18 reverse phase capillary column $(300 \mu \mathrm{m}$ inner diameter $\times 15 \mathrm{~cm}$; Agilent Technologies). The mobile phases were $0.1 \%$ formic acid (A), and $84 \% \mathrm{CH}_{3} \mathrm{CN}$ with $0.1 \%$ formic acid (B). The flow rate was $500 \mathrm{~nL} / \mathrm{min}$ with a linear gradient of $4-50 \%$ B over $50 \mathrm{~min}$, an increase to $100 \%$ B over $4 \mathrm{~min}$, then $100 \%$ B for $10 \mathrm{~min}$. The peaks were injected online into a Finnigan LTQ (single linear quadrupole ion trap) mass spectrometer for peptide identification. Mass spectrometry was performed on a Finnigan LTQ linear ion trap. The mass spectrometry (MS) method consisted of a cycle combining one full MS scan with two MS/MS events (25\% collision energy). The dynamic exclusion duration was set to $30 \mathrm{~s}$.

\section{Fluorescent localization of TTR and GRP78}

hRECs from passage 5 were incubated under normal conditions for $24 \mathrm{~h}$ at $37^{\circ} \mathrm{C}$ in 24 well plates, after which $4 \mu \mathrm{mol} / \mathrm{L} N$-(5-Fluoresceinyl) maleimide-labeled TTR was added to the media, and the cells were incubated for 1-6 h longer. After this incubation, the wells were washed 6 times with the primary media, and the cells were then visualized using a fluorescence microscope (Olympus Corp, Tokyo, Japan).

After culturing hRECs (from passage 5) in normal media for $24 \mathrm{~h}$ at $37^{\circ} \mathrm{C}$, approximately $4 \mu \mathrm{mol} / \mathrm{L}$ TTR (unlabeled) was added for $6 \mathrm{~h}$. The cells in wells were washed three times with PBS, fixed in $4 \%$ paraformaldehyde for 20 minutes, permeabilized in $0.1 \%$ Triton $\mathrm{X}-100$, and exposed to the indicated primary antibodies overnight at $4{ }^{\circ} \mathrm{C}$. After exposure to secondary antibodies and counterstaining with Hoechst33342, the cells were visualized using a fluorescence microscope (Olympus Corp, Tokyo, Japan).

\section{Cell transfection and GRP78 RNAi assays}

hRECs (from passage 6) were transiently transfected with $100 \mathrm{nmol} / \mathrm{L}$ of GRP78 siRNA (Santa Cruz Biotechnology, Santa Cruz, CA,USA) using Lipofectamine-2000 reagent (Invitrogen, Carlsbad, CA, USA) according to manufacturer's protocol. After incubation for $24 \mathrm{~h}$, hRECs were harvested for further experiments.

\section{Western blotting}

hRECs were harvested from 6-well plates, and the protein was extracted from the cells using RIPA buffer with protease inhibitors. The protein concentration was measured using a BCA assay kit. Equal amounts of protein ( $15 \mu \mathrm{L}$ of protein sample, $\sim 2 \mathrm{mg} / \mathrm{mL})$ were separated using 10\% SDS-PAGE and transferred to a nitrocellulose membrane. After blocking with 5\% non-fat-dried milk, the membrane was incubated with diluted primary antibodies overnight at room temperature $\left(\sim 25^{\circ} \mathrm{C}\right)$, followed by incubation with the secondary antibody. Western blotting was performed using antibodies against GRP78, PERK, CHOP, ATF4, eIF2 $\alpha$ and GAPDH. The quantitative densitometric values for each protein were normalized to GAPDH, and membranes were then scanned using an Odyssey Image System (LI-COR Biosciences, Lincoln, NE, USA). The band intensities were analyzed using Bio-Rad Quantity One software (version 4.4.0; Bio-Rad, Hercules, CA, USA) for quantification.

\section{Statistical analysis}

SPSS 13.0 for windows (Chicago, IL, USA) was used for data analysis, and statistical significance was determined using a T test. The $\mathrm{P}$ and $\mathrm{T}$ values were calculated. $\mathrm{P}<0.05$ was considered statistically significant.

\section{Results}

Production and fluorescent labeling of TTR

After the Ni-column separation, endotoxin depletion and fluorescent labeling, reducing SDS-PAGE analysis revealed a single band of the purified and tagged TTR below $20 \mathrm{kDa}$ as the monomer (Fig. 1A). Furthermore, on the basis of the optimal fluorescent signal of $\mathrm{N}-(5$ - 

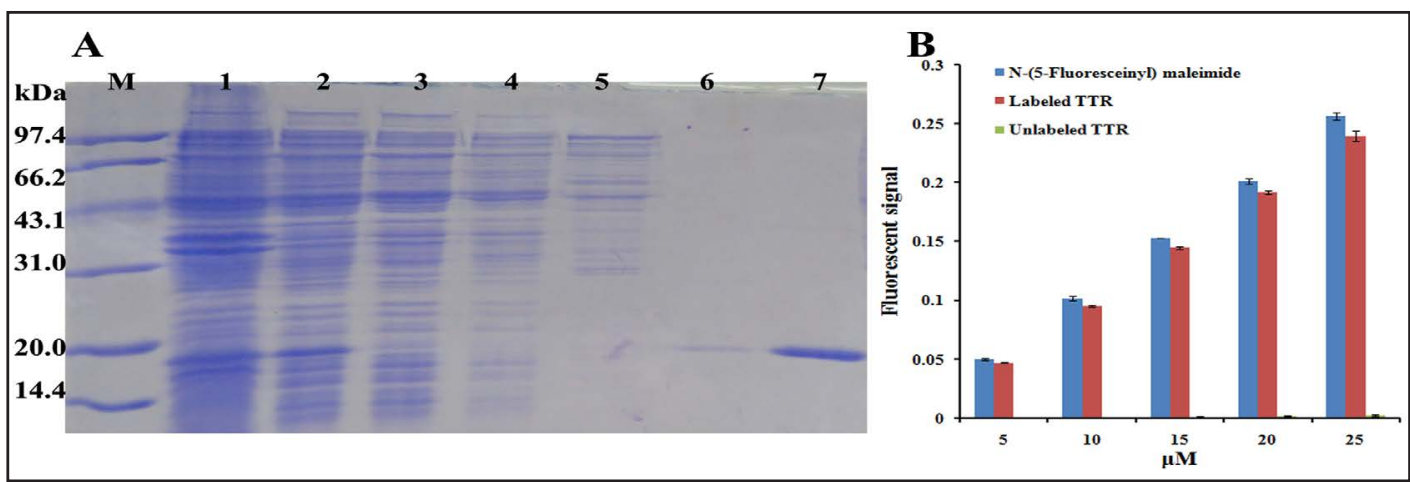

Fig. 1. Preparation and fluorescent labeling of human TTR (A) SDS-PAGE analysis of purified and labeled TTR. Lane M, the molecular mass marker; Lane 1, the whole cell proteins after sonication; Lane 2, the supernatant proteins after sonication; Lane 3, the flow-through proteins of the Ni-column; Lane 4, the proteins washed with $20 \mathrm{mM}$ imidazole; Lane 5, the proteins washed with $100 \mathrm{mM}$ imidazole; Lane 6, TTR purified in elution with $200 \mathrm{mM}$ imidazole; Lane 7, after eduotoxin was removed, TTR was site-directly labeled with N-(5-Fluoresceinyl) maleimide. The unlabeled and labeled TTRs all showed a single band below 20 $\mathrm{kDa}$ as monomer. (B) The labeling ratio was calculated as $\sim 95 \%$ owing to the fluorescent signal of the tag $\left(\lambda_{\mathrm{ex}} 492 \mathrm{~nm} ; \lambda_{\mathrm{em}} 518 \mathrm{~nm}\right)$.

Fluoresceinyl) maleimide $\left(\lambda_{\text {ex }} 492 \mathrm{~nm} ; \lambda_{\text {em }} 518 \mathrm{~nm}\right.$ ), the labeling ratio was calculated as $\sim 95 \%$ (n=3) (Fig. 1B).

TTR represses neovascularization in DR by inducing apoptosis

Our previous research showed that TTR inhibited the proliferation of hRECs and neovascularization $[13,14]$. To evaluate whether the decrease in cell proliferation induced by TTR was apoptotic, flow cytometry was used to detect the presence of cell death. In simulated DR environments with high glucose and hypoxia, we found that apoptosis occurred in about $30 \%$ of the cells treated with the combination of TTR and hypoxia, but apoptosis was not significantly different in the HG or normal environments (Fig. 2). This suggested that TTR significantly enhanced the anti-angiogenic effect by inducing apoptosis under hypoxic conditions ( $\mathrm{LG}+$ Hypoxia vs $\mathrm{LG}+$ Hypoxia+TTR, $\mathrm{p}=0.002$, T= $-24.844 ; \mathrm{HG}+$ Hypoxia vs $\mathrm{HG}+$ Hypoxia+TTR, $\mathrm{p}=0.002, \mathrm{~T}=-24.512$ ).

TTR affects hRECs by passing through the cell membrane to the nucleus

According to the fluorescent signal of $\mathrm{N}$-(5-Fluoresceinyl) maleimide tag that remained in the cells, TTR could enter hRECs within $6 \mathrm{~h}$ (Fig. 3A).

To investigate the exact location of the TTR in the hRECs, after incubation with TTR for 6 $h$, the coverslips were sequentially incubated with mouse anti-human TTR antibody and with Alexa Flour 488-conjugated donkey anti-mouse IgG secondary antibody. In the subsequent fluorescent analysis, a higher magnification of a same nucleus (marked with arrow) is shown in the upper right corner of each figure (Fig. 3). The nuclei were stained with Hoechst 33342 (Fig. 3B), and the TTR remaining in cells was stained with Alexa Flour 488 (Fig. 3C). The TTR region generally overlapped with nucleus (Fig. 3D). This revealed that TTR could efficiently cross the cell membrane and nuclear membrane.

\section{Identification of TTR-interacting proteins on membrane structures}

The TTR-captured membrane proteins were identified by LTQ-MS analysis. The MS/MS spectra acquired from all the runs were searched against the IPI HUMAN v3.87 database using the program SEQUEST. The SEQUEST filter was set to Xcorr $\geq 1.9$ for Charge +1 , Xcorr $\geq 2.2$ for Charge +2 , Xcorr $\geq 3.75$ for Charge +3 , and DelCN $\geq 0.1$. Thirty unique proteins with more than four peptides and more than 15\% CoverPercent were identified (Table 2). GRP78 was one of the most abundant of the TTR-captured proteins. 


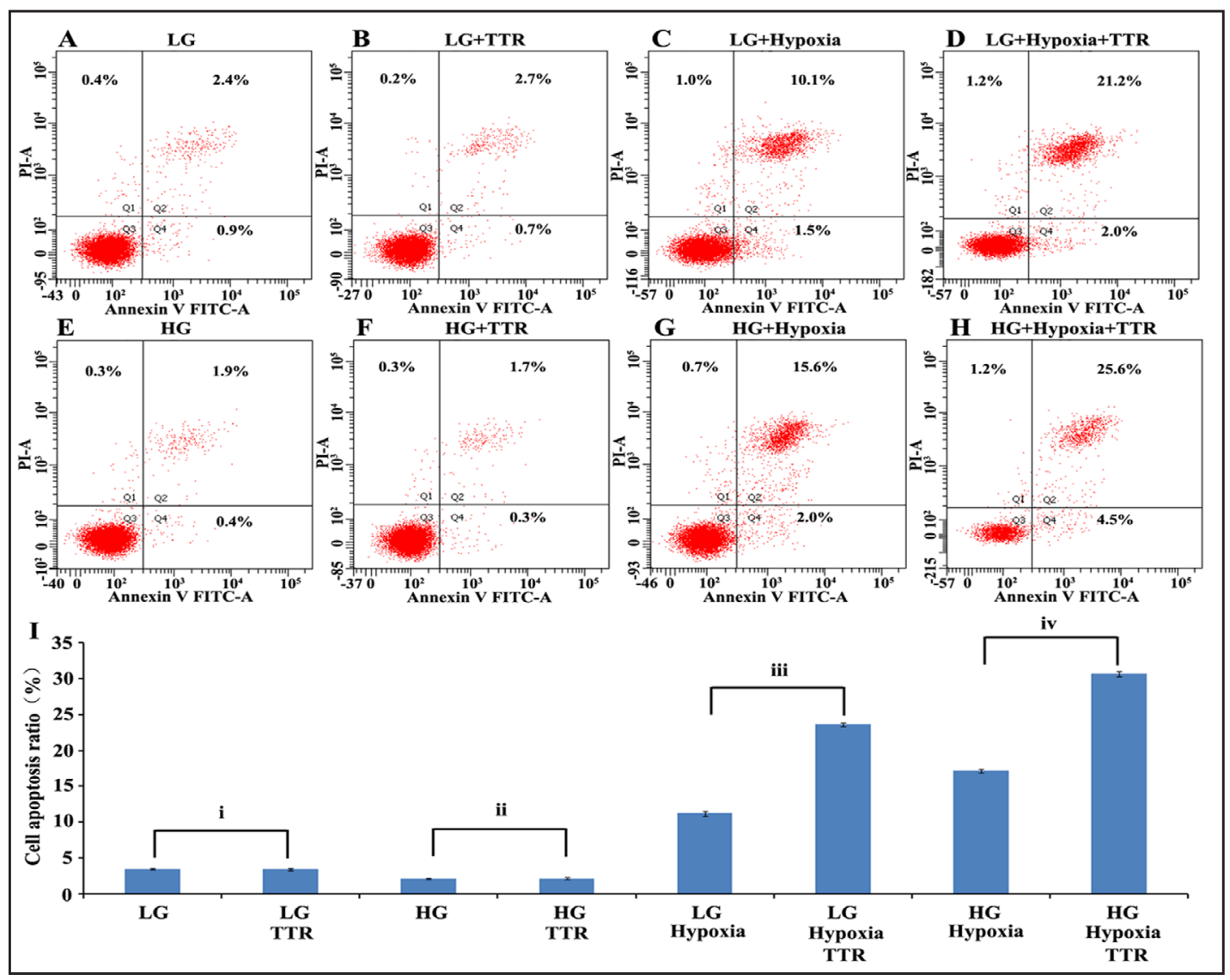

Fig. 2. TTR affects the apoptosis of hRECs Flow cytometric analysis of hRECs for apoptosis in (A) LG media; (B) LG media with TTR; (C) LG media with hypoxia; (D) LG media with hypoxia and TTR; (E) HG media; (F) HG media with TTR; (G) HG media with hypoxia; (H) HG media with hypoxia and TTR; (I) As calculated, in media without hypoxia, apoptosis of hRECs could not be regulated by exogenous TTR (i: LG vs LG+TTR, $\mathrm{T}<-0.001, \mathrm{p}=1.000$; ii: HG vs HG+TTR, T= -0.329, $\mathrm{p}=0.774$ ). However, in media with hypoxia, the apoptosis ratio of hRECs were significantly enhanced by exogenous TTR (iii: LG+hypoxia vs LG+hypoxia+TTR, T= -24.844, p= 0.002; iv: HG+hypoxia vs HG+hypoxia+TTR, T= -24.512, p=0.002).

Table 2. TTR-interacting proteins identified in LTQ/MS

\begin{tabular}{|c|c|c|c|c|c|}
\hline No. & Protein & UniquePepCount & CoverPercent (\%) & Theoretical Mw (Da) & PI \\
\hline 1 & CH60_HUMAN 60 kDa heat shock protein & 17 & $40.84 \%$ & 61053.94 & 5.7 \\
\hline 2 & GRP78_HUMAN 78 kDa glucose-regulated protein & 13 & $24.62 \%$ & 72332.07 & 5.07 \\
\hline 3 & BOYJC4_HUMAN Vimentin & 13 & $31.79 \%$ & 49652.81 & 5.19 \\
\hline 4 & B4E335_HUMAN cDNA FLJ52842, highly similar to Actin & 11 & $42.74 \%$ & 39225.52 & 5.39 \\
\hline 5 & I6L965_HUMAN KRT18 protein & 11 & $48.39 \%$ & 42172.83 & 5.01 \\
\hline 6 & ATPB_HUMAN ATP synthase subunit beta & 11 & $39.32 \%$ & 56559.22 & 5.26 \\
\hline 7 & ENPL_HUMAN Endoplasmin & 10 & $15.09 \%$ & 92338.65 & 4.77 \\
\hline 8 & B7Z4V2_HUMAN cDNA FLJ51907, highly similar to Stress-70 protein & 10 & $21.80 \%$ & 72400.2 & 5.7 \\
\hline 9 & ACTN1_HUMAN Alpha-actinin-1 & 8 & $15.13 \%$ & 103056.37 & 5.25 \\
\hline 10 & G3P_HUMAN Glyceraldehyde-3-phosphate dehydrogenase & 8 & $28.36 \%$ & 36048.8 & 8.57 \\
\hline 11 & H7BZ94_HUMAN Protein disulfide-isomerase & 7 & $18.97 \%$ & 52501.53 & 4.76 \\
\hline 12 & HUMAN cDNA FLJ51028, highly similar to $60 \mathrm{kDa}$ heat shock protein & 6 & $35.10 \%$ & 26685.36 & 5.12 \\
\hline 13 & Q6IPN6_HUMAN Elongation factor 1-alpha & 6 & $18.40 \%$ & 50122.24 & 9.1 \\
\hline 14 & V9HW96_HUMAN Chaperonin containing TCP1, subunit 2 (Beta) & 6 & $21.50 \%$ & 57487.56 & 6.01 \\
\hline 15 & B4DRT3_HUMAN Pyruvate kinase & 6 & $16.44 \%$ & 55925.94 & 7.2 \\
\hline 16 & E9PNE6_HUMAN Heat shock cognate $71 \mathrm{kDa}$ protein & 6 & $18.00 \%$ & 54975.39 & 5.51 \\
\hline 17 & ACTH_HUMAN Actin, gamma-enteric smooth muscle & 6 & $19.15 \%$ & 41876.43 & 5.31 \\
\hline 18 & TBA1C_HUMAN Tubulin alpha-1C chain & 6 & $23.61 \%$ & 49894.78 & 4.96 \\
\hline 19 & Q53G71_HUMAN Calreticulin variant & 5 & $23.74 \%$ & 48140.96 & 4.29 \\
\hline 20 & Q53HV2_HUMAN Chaperonin containing TCP1, subunit 7 (Eta) variant & 5 & $19.34 \%$ & 59339.88 & 7.55 \\
\hline 21 & HOYN42_HUMAN Annexin & 5 & $17.11 \%$ & 38575.54 & 7.57 \\
\hline 22 & FUMH_HUMAN Fumarate hydratase & 5 & $21.37 \%$ & 54636.39 & 8.85 \\
\hline 23 & B3KT06_HUMAN cDNA FLJ37398 fis, clone BRAMY2027467, highly similar to Tubulin alpha-ubiquitous chain & 5 & $19.71 \%$ & 46340.8 & 5 \\
\hline 24 & ENOA_HUMAN Alpha-enolase & 5 & $23.96 \%$ & 47168.41 & 7.01 \\
\hline 25 & RAB7A_HUMĀN Ras-related protein Rab-7a & 5 & $34.78 \%$ & 23489.48 & 6.4 \\
\hline 26 & HNRPK_HUMAN Heterogeneous nuclear ribonucleoprotein $\mathrm{K}$ & 5 & $18.36 \%$ & 50975.66 & 5.39 \\
\hline 27 & CH10_HUMAN $10 \mathrm{kDa}$ heat shock protein & 4 & $43.14 \%$ & 10931.55 & 8.89 \\
\hline 28 & RA1L2_HUMAN Heterogeneous nuclear ribonucleoprotein A1-like 2 & 4 & $15.63 \%$ & 34224.94 & 9.08 \\
\hline 29 & B4DPJ8_HUMAN cDNA FLJ52344, highly similar to T-complex protein 1 subunit zeta & 4 & $14.31 \%$ & 58023.51 & 6.24 \\
\hline 30 & E9PCA1_HUMAN T-complex protein 1 subunit epsilon & 4 & $15.90 \%$ & 59670.4 & 5.45 \\
\hline
\end{tabular}




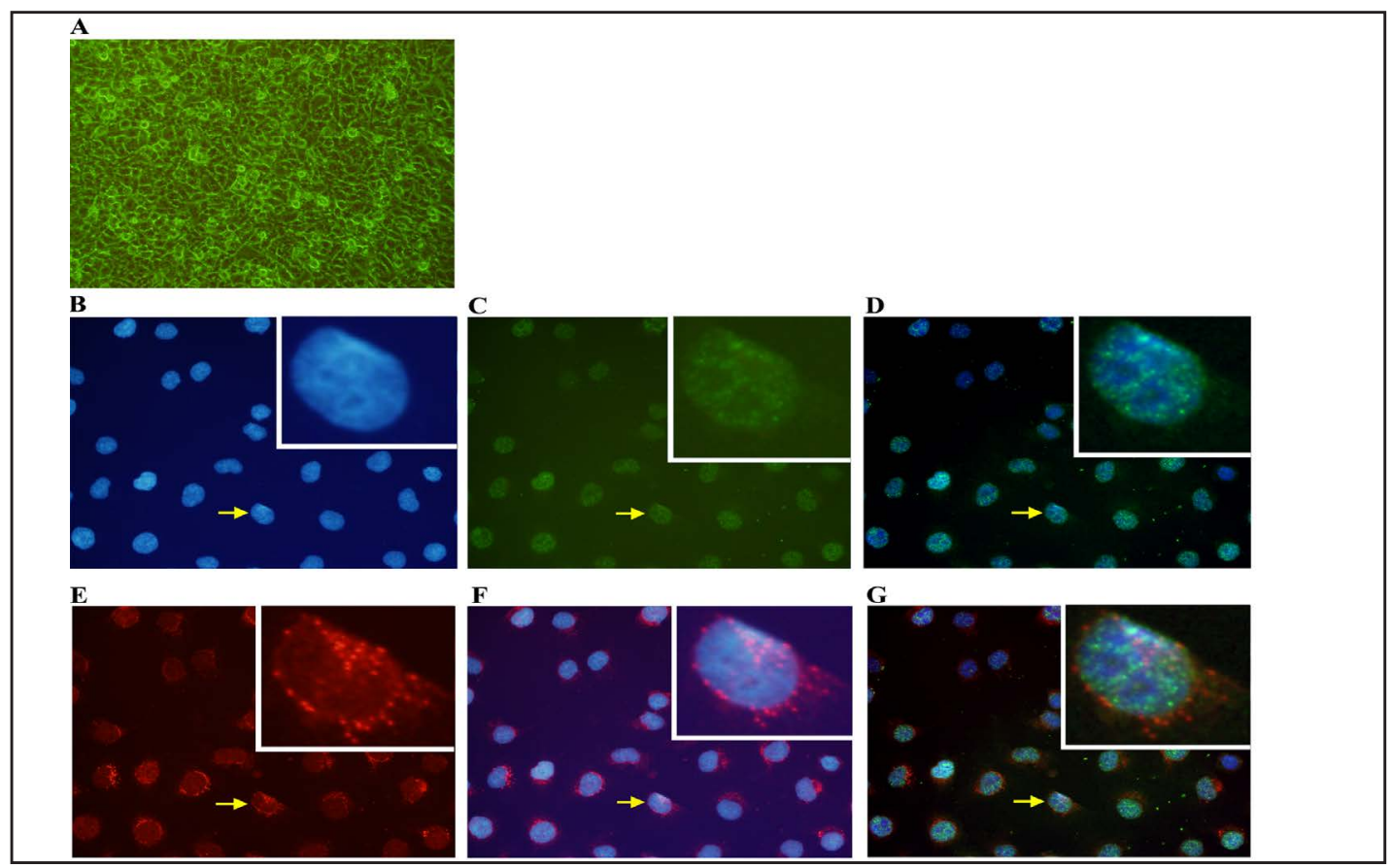

Fig. 3. TTR and GRP78 partially overlap in and around the nuclei of hRECs(A) The fluorescent signal of N-(5-Fluoresceinyl) maleimide tag showed that TTR could efficiently enter hRECs. (B)-(G) A higher magnification of a same nucleus (marked with arrow) is shown in the upper right corner of each Fig.. (B) The nucleus was counterstained with Hoechst 33342 as control; (C) TTR in hRECs was recognized by mouse anti-human TTR antibody and Alexa Flour 488 labeled donkey anti-mouse IgG antibody; (D) TTR region mainly covered the nucleus (E) GRP78 in hRECs was recognized by rabbit anti-human GRP78 antibody and Alexa Flour 594 labeled donkey anti-rabbit IgG antibody; (F) GRP78 region was detected as in and around the nucleus (G) The locations of TTR and GRP78 partially overlapped in and around the nuclei.

Fig. 4. The effect of TTR on the expression of GRP78 and proteins in eIF2 $\alpha$ / CHOP pathwayGAPDH was used as a control. (A) In hypoxia media, TTR upregulated the expression of GRP78 (in Line GRP78, Lane 3 vs 4 and Lane 7 vs 8 ), eIF $2 \alpha$ (in Line eIF $2 \alpha$, Lane 3 vs 4 and Lane 7 vs 8 ), PERK (in Line PERK, Lane 3 vs 4 and Lane 7 vs 8), ATF4 (in Line ATF4, Lane 3 vs 4 and Lane 7 vs 8 ) and CHOP in Line CHOP, Lane 3 vs 4 and Lane 7 vs 8); (B) After hRECs cells were transfected with GRP78 siRNA, the expression of GRP78 was inhibited, and the levels of eIF $2 \alpha$, PERK, ATF4 and CHOP were subsequently decreased.

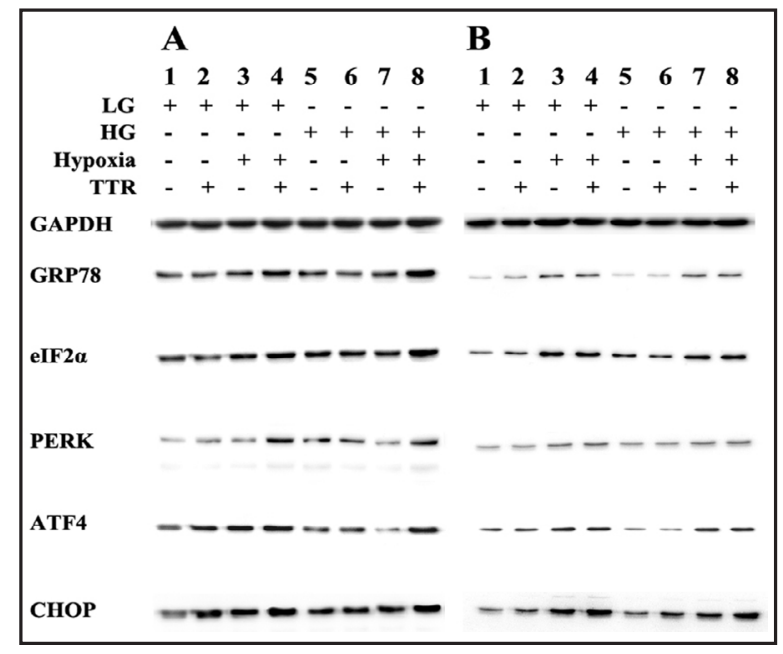

The location of GRP78 in hRECs was investigated using immunofluorescence staining. The coverslips with the cultured cells were sequentially incubated with rabbit anti-human GRP78 antibody and with Alexa Flour 594-conjugated donkey anti-rabbit IgG secondary antibody. The nuclear regions were stained with Hoechst 33342 (Fig. 3B), and the GRP78 region was stained with Alexa Flour 594 (Fig. 3E). The locations of TTR and GRP78 partially overlapped in and around the nuclei (Fig. 3F, G).

These results suggested that TTR might directly interact with GRP78.

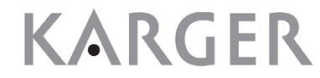




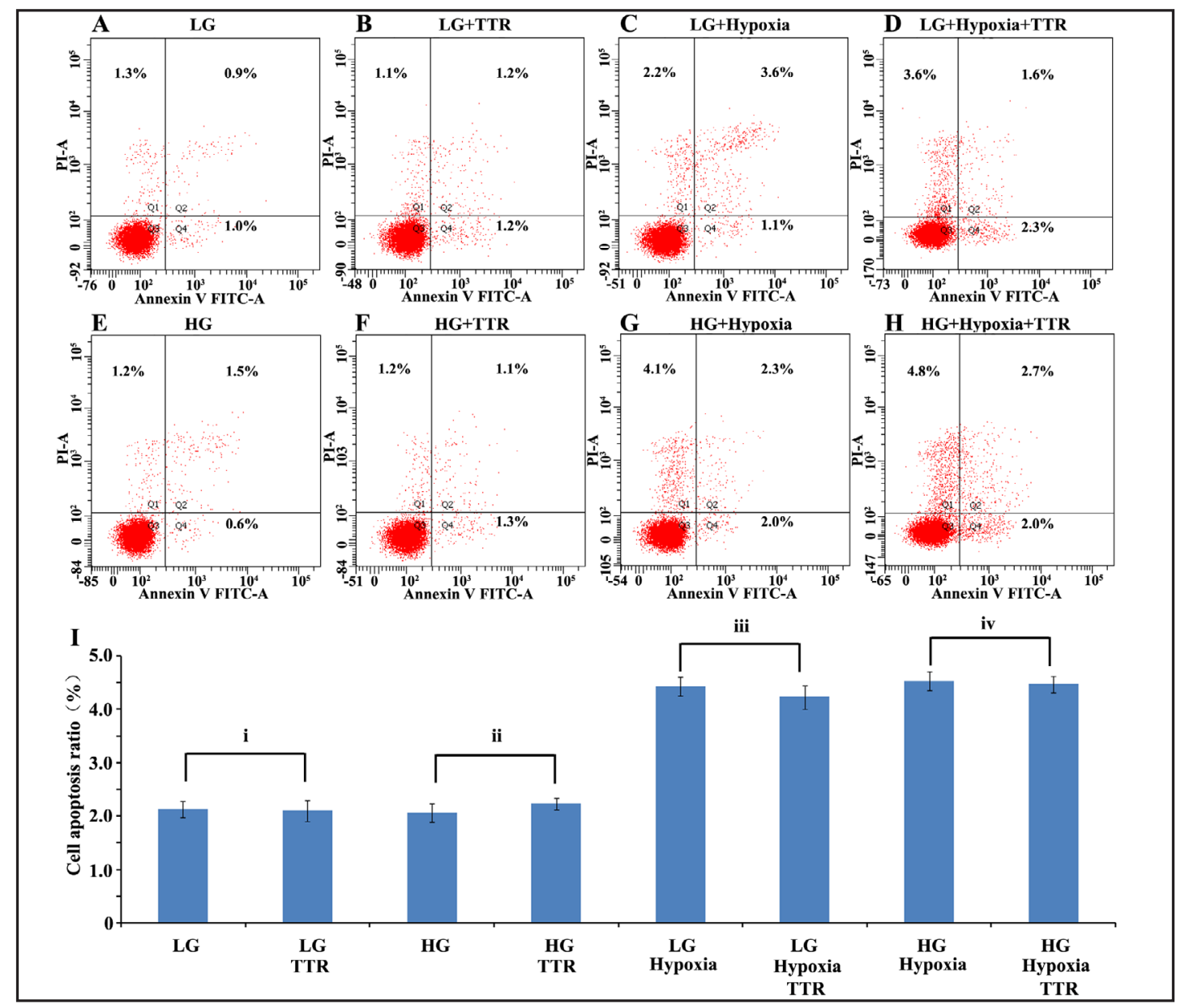

Fig. 5. GRP78 siRNA blocks the effect of TTR on the apoptosis of hRECsAfter transfected with GRP78 siRNA, flow cytometric analysis of hRECs for apoptosis in (A) LG media; (B) LG media with TTR; (C) LG media with hypoxia; (D) LG media with hypoxia and TTR; (E) HG media; (F) HG media with TTR; (G) HG media with hypoxia; (H) HG media with hypoxia and TTR; (I) As calculated, after GRP78 RNAi, in all media (including LG, LG+Hypoxia, HG and HG+Hypoxia), apoptosis of hRECs could not be significantly regulated by exogenous TTR any more (i: LG vs LG+TTR, T= 0.125, p= 0.912; ii: HG vs HG+TTR, T= -1.250, p= 0.388; iii: $L G+$ hypoxia vs $\mathrm{LG}+$ hypoxia+TTR, T=0.667, p= 0.574; iv: HG+hypoxia vs HG+hypoxia+TTR, T=0.256, p=0.822).

TTR upregulates GRP78 and triggers cell transition from ER stress to apoptosis through EIF2 $\alpha / C H O P$ pathway

To further examine the relationship between TTR and GRP78, the GRP78 contents were evaluated using western blotting assays. Consistent with LTQ-MS data, we observed a noticeable upregulation of GRP78 in the cells that had been treated with TTR combined with hypoxia (Fig. 4A, in Line GRP78, Lane 3 vs 4 and Lane 7 vs 8).

Eukaryotic translationinitiation factor alpha (eIF2 $\alpha$ ), protein kinase RNA-like endoplasmic reticulum kinase (PERK) and activating transcription factor-4 (ATF4) have been reported to constitute the essential pathway underlying the unfolded protein response (UPR) [26-28]. These proteins are closely associated with GRP78 signaling pathways. In western blots (Fig. 4A), eIF $2 \alpha$ (in Line eIF2 $\alpha$, Lane 3 vs 4 and Lane 7 vs 8), PERK (in Line PERK, Lane 3 vs 4 and Lane 7 vs 8) and ATF4 (in Line ATF4, Lane 3 vs 4 and Lane 7 vs 8) were increased by the combination of TTR and hypoxia. Furthermore, enhanced expression of C/EBP homologous protein (CHOP) was observed in cells that had been treated with the combination of TTR and hypoxia (Fig. 4A, in Line CHOP, Lane 3 vs 4 and Lane 7 vs 8). Together, 


\section{Cellular Physiology Cell Physiol Biochem 2017;43:788-800

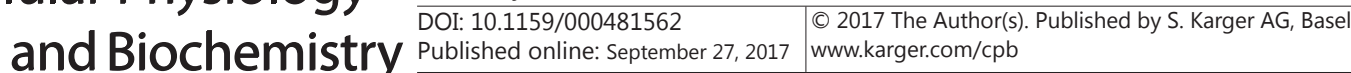 \\ Shao et al.: TTR Induces HRECs Apoptosis Through GRP78-Pathway in DR}

these results suggested that TTR caused an upregulation of GRP78 in hypoxia, which led to the progression from ER stress to apoptosis through eIF $2 \alpha / \mathrm{CHOP}$ pathway.

\section{GRP78 siRNA partially reverses TTR-induced apoptosis in hRECS}

For the rescue experiments, the viability of hRECs that had been transfected with GRP78 siRNA was investigated. GRP78 siRNA partially reversed the TTR-induced apoptosis of hRECs in the simulated DR environments (Fig. 5). The expression of GRP78 after the transient transfection of siRNA is decreased (Fig. 4A and B, in Line GRP78). To investigate whether the downregulation of PERK, eIF2 $\alpha$, ATF4 and CHOP was dependent on GRP78, expression of these proteins was compared in TTR/ low glucose/high glucose $/ \mathrm{CoCl}_{2}$-treated cells with and without GRP78 siRNA. In the cells treated with the RNAi, the expression levels of PERK (Fig. 4 A and B, in Line PERK), eIF2 $\alpha$ (Fig. 4 A and B, in Line eIF2 $\alpha$ ), ATF4 (Fig. 4 A and B, in Line ATF4) and CHOP (Fig. 4 A and B, in Line CHOP) revealed reduced trends. Taken together, our results revealed that TTR facilitated apoptosis through a GRP78-dependent pathway.

\section{Discussion}

TTR is mainly secreted by the hRPECs and choroid in the eyes; ocular diseases such as high myopia and vitreous amyloidosis are associated with alterations in the structure or function of TTR $[29,30]$. Our previous study showed that only in a high glucose environment, but independent of hypoxia, could TTR (exogenous and endogenous) repress the proliferation, migration and tube formation by regulating the key genes in the Tie2 pathway [18]. In this study, we found that TTR significantly induced apoptosis in hypoxic environments, regardless of hyperglycemia. These phenomena suggested that, in the DR environment, hypoxia was necessary for the TTR-mediated apoptosis of the hRECs, whereas hyperglycemia was required for TTR to inhibit the hRECs proliferation.

A group of TTR-captured membrane proteins were identified with LTQ-MS analysis, and GRP78 was revealed to be one of the most abundant. GRP78 (BiP) is a 78-kDa protein chaperone $[26,27]$ that has been proven to prevent the cell death caused by disturbance of endoplasmic reticulum (ER) homeostasis [28] and to mediate essential components of ER stress-induced apoptosis [31-33]. Fluorescent labeling and immunofluorescence staining indicated that TTR efficiently crossed cell membrane and nuclear membrane; the localization of TTR and GRP78 partially overlapped in and around the nuclei. In rescue experiments, GRP78 siRNA partially prevented the TTR-induced apoptosis. These results suggested that there might be a direct interaction between TTR and GRP78. Furthermore, in the hypoxic DR environment, TTR might target GRP78 to trigger cell apoptosis. Here, our results show for the first time that TTR upregulates GRP78 to control cell apoptosis.

The ER is the major organelle responsible for protein folding, lipid biosynthesis and $\mathrm{Ca}^{2+}$ storage. Recent studies have established that the ER also functions as an intracellular signaling platform that regulates cell fate and activity through the unfolded protein response (UPR) [34]. With ER stress, three ER transmembrane proteins activate the UPR: PERK, ATF 4 and eIF2 $\alpha$. These UPR branches coordinate the regulation of protein translation and transcription programs to restore ER homeostasis [35]. Unresolved ER stress activates proapoptotic and proinflammatory genes, such as those encoding CHOP, which contributes to retinal inflammation and vascular dysfunction in ischemic retinopathy and DR [36]. In endothelial cells, activation of the UPR plays a critical role in regulating cell proliferation, apoptosis and angiogenesis [37], and in this pathway, CHOP is a well-characterized marker for the cell transition from ER stress to apoptosis [38, 39]. Recent research has provided the first investigation of a role for ER stress in the dysfunction of angiogenic progenitors in diabetes, and the GRP78-mediated pathway is the key step [40]. In our research, we observed that TTR meaningfully increased CHOP and enhanced the expression of eIF2 $\alpha$, PERK and ATF4. Together, these data suggested that TTR might cause ER stress and eventually trigger the cell transition from ER stress to apoptosis.

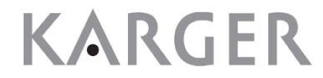




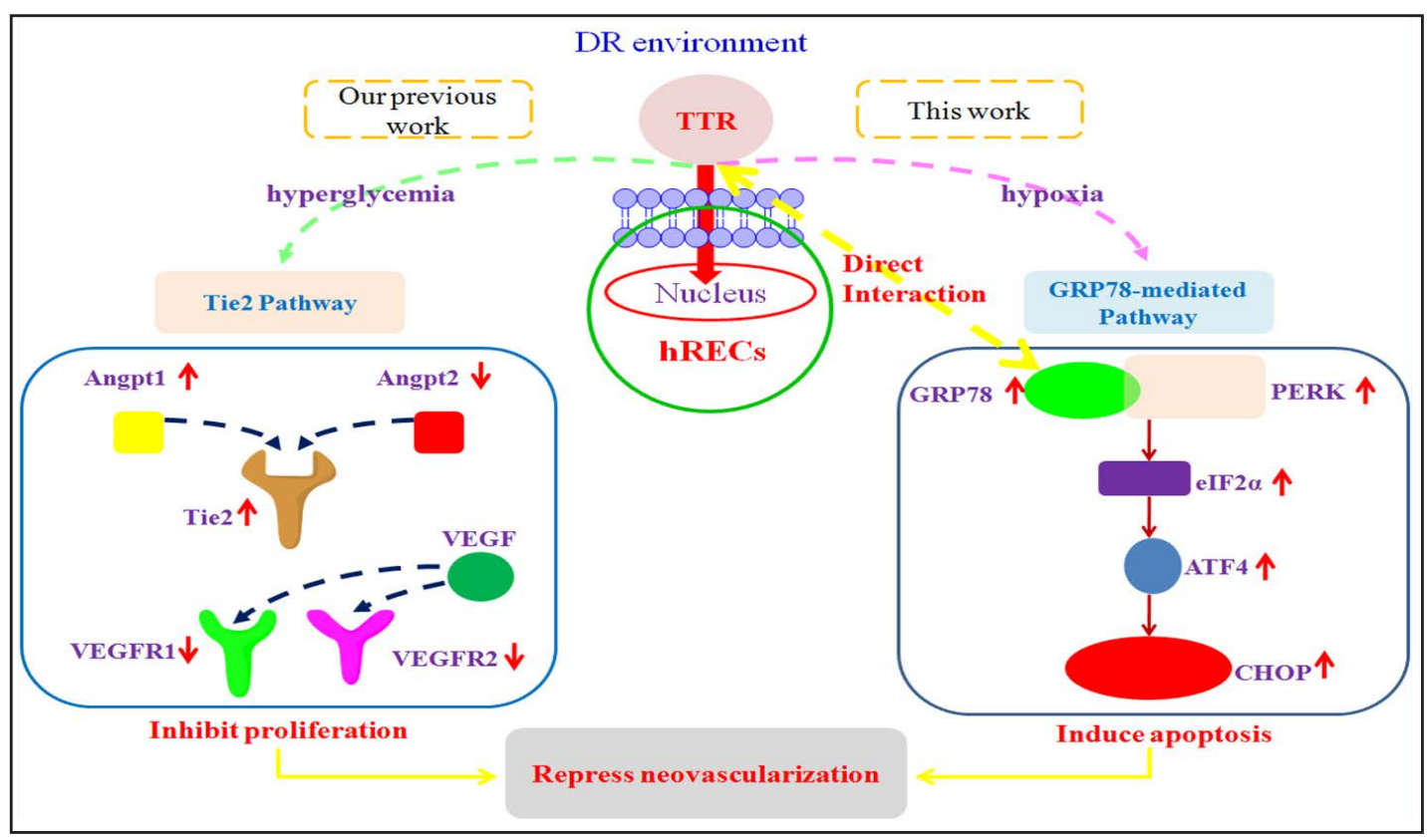

Fig. 6. Schematic overview of the TTR-mediated neovascularization repression in DR environmentTTR passes through cell membrane to nucleus of hRECs, and it might directly interact with GRP78; TTR induces apoptosis of hRECs through GRP78-mediated Pathway in hypoxic environment; TTR inhibits proliferation of hRECs through Tie2 Pathway in hyperglycemic environment.

\section{Conclusion}

In a DR environment with hypoxia, TTR could induce the apoptosis of hRECs through a GRP78-dependent pathway by regulating the expression of GRP78, eIF2 $\alpha$, PERK, ATF4 and CHOP. On the other hand, according to our previous work [18], hyperglycemia in the DR environment should be necessary for TTR to inhibit the proliferation, migration and tube formation of hRECs by affecting the Tie2 pathway. However, the details of these processes and the mechanisms by which TTR could enter the nucleus are still not clear (Fig. 6). To understand the mechanism of these phenomenon more thoroughly, future studies should consider performing gene chip analysis and target molecule screening analysis to integrate the whole pathway. For clinical applications, the serum and vitreous TTR levels in DR development will require systematic investigation, and these results may help to refine the grading and diagnosis of DR. In addition, for the prevention and therapy of DR, maintaining a relatively high TTR level in ocular tissue might be considered a possible future approach.

\section{Acknowledgements}

This work was supported by National Natural Science Foundation of China (Grant No. 81400415), Natural Science Foundation of Jiangsu Province (BK20160053 and BK20151105), Youth Medical Talent Project of Jiangsu Province (QNRC2016182) and Project of Hospital Management Center of Wuxi (YGZXM1509).

\section{Disclosure Statement}

The authors declare no conflict of interest. 


\section{Cellular Physiology Cell Physiol Biochem 2017;43:788-800 \begin{tabular}{l|l|l} 
and Biochemistry Published online: September 27, 2017 & $\begin{array}{l}\text { (c) } 2017 \text { The Author(s). Published by S. Karger AG, Basel } \\
\text { www.karger.com/cpb }\end{array}$
\end{tabular}}

\section{References}

1 Madonna R, Balistreri CR, Geng YJ, De Caterina R: Diabetic microangiopathy: Pathogenetic insights and novel therapeutic approaches. Vascul Pharmacol 2017;90:1-7.

-2 Liu Y, Song Y, Tao L, Qiu W, Lv H, Jiang X, Zhang M, Li X: Prevalence of diabetic retinopathy among 13473 patients with diabetes mellitus in China: a cross-sectional epidemiological survey in six provinces. BMJ Open 2017;7:e013199.

-3 Amin J, Sharif M, Yasmin M: A review on recent developments for detection of diabetic retinopathy. Scientifica (Cairo) 2016;6838976.

-4 Parikh RN, Traband A, Kolomeyer AM, VanderBeek BL, Kim BJ, Maguire AM, Brucker AJ: Intravitreal bevacizumab for the treatment of vitreous hemorrhage due to proliferative diabetic retinopathy. Am J Ophthalmol 2017;176:194-202.

5 Veluthakal R, Kumar B, Mohammad G, Kowluru A, Kowluru RA: Tiam1-Rac1 axis promotes activation of p38 MAP kinase in the development of diabetic retinopathy: Evidence for a requisite role for protein palmitoylation. Cell Physiol Biochem 2015;36:208-220.

6 Li YJ, Jiang Q, Cao GF, Yao J, Yan B: Repertoires of autophagy in the pathogenesis of ocular diseases. Cell Physiol Biochem 2015;35:1663-1676.

7 Ishimoto Y, Hirota-Takahata Y, Kurosawa E, Chiba J, Iwadate Y, Onozawa Y, Hasegawa T, Tamura A, Tanaka M, Kobayashi H: A novel natural product-derived compound, Vestaine A1, exerts both pro-angiogenic and anti-permeability activity via a different pathway from VEGF. Cell Physiol Biochem 2016;39:1905-1918.

$>8$ Li J, Wang P, Ying J, Chen Z, Yu S: Curcumin attenuates retinal vascular leakage by inhibiting calcium/ calmodulin-dependent protein kinase II activity in streptozotocin-induced diabetes. Cell Physiol Biochem 2016;39:1196-208.

-9 Campochiaro PA, Peters KG: Targeting Tie2 for treatment of diabetic retinopathy and diabetic macular edema. Curr Diab Rep 2016;16:126.

-10 Mammadzada P, Gudmundsson J, Kvanta A, André H: Differential hypoxic response of human choroidal and retinal endothelial cells proposes tissue heterogeneity of ocular angiogenesis. Acta Ophthalmol 2016;94:805-814.

11 Wu J, Ke X, Ma N, Wang W, Fu W, Zhang H, Zhao M, Gao X, Hao X, Zhang Z: Formononetin, an active compound of Astragalus membranaceus (Fisch) Bunge, inhibits hypoxia-induced retinal neovascularization via the HIF-1 $\alpha$ /VEGF signaling pathway. Drug Des Devel Ther 2016;10:3071-3081.

12 Wu J, Ke X, Wang W, Zhang H, Ma N, Fu W, Zhao M, Gao X, Hao X, Zhang Z: Aloe-emodin suppresses hypoxiainduced retinal angiogenesis via inhibition of HIF-1 $\alpha$ /VEGF pathway. Int J Biol Sci 2016;12:1363-1371.

13 Monaco HL, Rizzi M, Coda A: Structure of a complex of two plasma proteins: transthyretin and retinolbinding protein. Science 1995;268:1039-1041.

$\checkmark 14$ Delcourt C, Dupuy AM, Carriere I, Lacroux A, Cristol JP: Albumin and transthyretinas risk factors for cataract: the POLA study. Arch Ophthalmol 2005;123:225-232.

15 Mullins RF, Russell SR, Anderson DH, Hageman GS: Drusen associated with aging and age-related macular degeneration contain proteins common to extracellular deposits associated with atherosclerosis, elastosis, amyloidosis, and dense deposit disease. FASEB J 2000;14:835-846.

-16 Shao, J, Xin, Y, Yao Y: Correlation of misfolded transthyretin in abnormal vitreous and high myopia related ocular pathologies. Clin Chim Acta 2011;412:2117-2121.

17 Shao J, Xin, Y, Yao, Y, Zhu J: Functional analysis of misfolded transthyretin extracted from abnormal vitreous with high myopia related ocular pathologies. Clin Chim Acta 2013;415:20-24.

18 Shao J, Yao Y: Transthyretin represses neovascularization in diabetic retinopathy. Mol Vis 2016;22:11881197.

19 Shao J, Yao Y: Repression of retinal microvascular endothelial cells by transthyretin under diabetic retinopathy condition. Int J Ophthalmol 2016;7:809-815.

20 Steinmetz E: Expresso $®$ cloning and expression systems: Expressioneering ${ }^{\mathrm{TM}} \mathrm{Tech}$ nology streamlines recombinant protein expression. Nat Methods 2011;8:3-4.

-21 Smith MEB, Caspersen MB, Robinson E, Morais M, Maruani A, Nunes JPM, Nicholls K, Saxton MJ, Caddick $\mathrm{S}$, Baker JR, Chudasama V: A platform for efficient, thiol-stable conjugation to albumin's native single accessible cysteine. Org Biomol Chem 2015;13:7831-7832. 


\section{Cellular Physiology Cell Physiol Biochem 2017;43:788-800 and Biochemistry DOI: 10.1159/000481562 2017 (O 2017 The Author(s). Published by S. Karger AG, Basel

22 Mehtala JG, Kulczar C, Lavan M, Knipp G, Wei A: Cys34-PEGylated human serum albumin for drug binding and delivery. Bioconjug Chem 201;26:941-949.

-23 Hanson ES, Foot LM, Leibold EA: Hypoxia post-translationally activates iron-regulatory protein 2. J Biol Chem 1999;274:5047-5052.

-24 Caltana L, Merelli A, LazarowskiA, Brusco A: Neuronal and glial alterations due to focal cortical hypoxia induced by direct cobalt chloride $\left(\mathrm{CoCl}_{2}\right)$ brain injection. Neurotox Res 2009;15:348-358.

-25 Lin K, Ye P, Liu J, He F, Xu W: Endostar inhibits hypoxia-induced cell proliferation and migration via the hypoxia-inducible factor- $1 \alpha /$ vascular endothelial growth factor pathway in vitro. Mol Med Rep 2015;11:3780-3785.

-26 Lin J, Chung S, Ueda K, Matsuda K, Nakamura Y, Park JH: GALNT6 stabilizes GRP78 protein by O-glycosylation and enhances its activity to suppress apoptosis under stress condition. Neoplasia 2017;19:43-53.

27 Guo H, Cao A, Chu S, Wang Y, Zang Y, Mao X, Wang H, Wang Y, Liu C, Zhang X, Peng W: Astragaloside IV attenuates podocyte apoptosis mediated by endoplasmic reticulum stress through upregulating sarco/ endoplasmic reticulum $\mathrm{Ca}^{2+}$-ATPase 2 expression in diabetic nephropathy. Front Pharmacol 2016;7:500.

28 Chen Y, Gui D, Chen J, He D, Luo Y, Wang N: Down-regulation of PERK-ATF4-CHOP pathway by Astragaloside IV is associated with the inhibition of endoplasmic reticulum stress-induced podocyte apoptosis in diabetic rats. Cell Physiol Biochem 2014;33:1975-1987.

-29 Kerschen P, Planté-Bordeneuve V: Current and future treatment approaches in transthyretin familial amyloid polyneuropathy. Curr Treat Options Neurol 2016;18:53.

-30 Shao J, Xin Y, Li R, Fan Y: Vitreous and serum levels of transthyretin (TTR) in high myopia patients are correlated with ocular pathologies. Clin Biochem 2011;44:681-685.

-31 Wang ZS, Xiong F, Xie XH, Chen D, Pan JH, Cheng L: Astragaloside IV attenuates proteinuria in streptozotocin-induced diabetic nephropathy via the inhibition of endoplasmic reticulum stress. BMC Nephrol 2015;16:44.

32 Fu WM, Lu YF, Hu BG, Liang WC, Zhu X, Yang HD, Li G, Zhang JF: Long noncoding RNA Hotair mediated angiogenesis in nasopharyngeal carcinoma by direct and indirect signaling pathways. Oncotarget 2016;7:4712-4723.

-33 Yang HP, Fan SJ, An Y, Wang X, Pan Y, Xiaokaiti Y, Duan J, Li X, Tie L, Ye M, Li X: Bisdemethoxycurcumin exerts pro-apoptotic effects in human pancreatic adenocarcinoma cells through mitochondrial dysfunction and a GRP78-dependent pathway. Oncotarget 2016;7:83641-83656.

34 Tao TQ, Wang XR, Liu M, Xu FF, Liu XH: Myofibrillogenesis regulator-1 attenuated hypoxia/reoxygenationinduced apoptosis by inhibiting the PERK/Nrf2 pathway in neonatal rat cardiomyocytes. Apoptosis 2015;20:285-297.

-35 Visioli F, Wang Y, Alam GN, Ning Y, Rados PV, Nör JE, Polverini PJ: Glucose-regulated protein 78 (Grp78) confers chemoresistance to tumor endothelial cells under acidic stress. PLoS One 2014;9:e101053.

-36 Fu D, Yu JY, Yang S, Wu M, Hammad SM, Connell AR, Du M, Chen J, Lyons TJ: Survival or death: a dual role for autophagy in stress-induced pericyte loss in diabetic retinopathy. Diabetologia 2016;59:2251-2261.

-37 Dong D, Ni M, Li J, Xiong S, Ye W, Virrey JJ, Mao C, Ye R, Wang M, Pen L, Dubeau L, Groshen S, Hofman FM, Lee AS: Critical role of the stress chaperone GRP78/BiP in tumor proliferation, survival, and tumor angiogenesis in transgene-induced mammary tumor development. Cancer Res 2008;68:498-505.

-38 Bahar E, Lee GH, Bhattarai KR, Lee HY, Choi MK, Rashid HO, Kim JY, Chae HJ, Yoon H: Polyphenolic extract of euphorbia supina attenuates manganese-induced neurotoxicity by enhancing antioxidant activity through regulation of ER stress and ER stress-mediated apoptosis. Int J Mol Sci 2017;18:E300.

39 Tao YK, Yu PL, Bai YP, Yan ST, Zhao SP, Zhang GQ: Role of PERK/eIF2 $\alpha /$ CHOP endoplasmic reticulum stress pathway in oxidized low-density lipoprotein mediated induction of endothelial apoptosis. Biomed Environ Sci 2016;29:868-876.

40 Bhatta M, Ma JH, Wang JJ, Sakowski J, Zhang SX: Enhanced endoplasmic reticulum stress in bone marrow angiogenic progenitor cells in a mouse model of long-term experimental type 2 diabetes. Diabetologia 2015;58:2181-2190. 